\title{
La reconstrucción de las experiencias de lectura de niñas en condición de vulnerabilidad
}

Beatriz Adriana Salgado González, Mexicana

Maestra en Investigación de la Educación

ORCID https://orcid.org/0000-0002-5906-3845

ratunop@gmail.com

Instituto Superior de Ciencias de la Educación del Estado de México

Recibido: 28 de junio del 2020

Aceptado: 03 de febrero del 2020

\section{Resumen}

En este artículo exponemos la reconstrucción de las experiencias de lectura de niñas en condición de vulnerabilidad que habitan en una Casa Hogar de la ciudad de Toluca considerado como un "Refugio", dadas sus desigualdades sociales, este tipo de contexto en el que habitan las niñas repercute en la construcción y reconstrucción de sí mismas. Y hablar de "sí mismo", de su vida, requiere "establecer un orden en el conjunto de hechos pasados, entre lo que era y es hoy, entre las experiencias pasadas y la valoración que han adquirido" (Bolívar, Domingo y Fernández, 2001, p. 43). Se trata de ir entramando una secuencia escrita de los acontecimientos que integran sus experiencias con la lectura, en ese sentido, podrán contar relatos desde su propia vida. Para la investigación, consideramos utilizar el enfoque narrativo interpretativo que nos permite, por medio de la reconstrucción de relatos dar cuenta del objeto de investigación. Teniendo presente que, a través de la narrativa, daremos sentido a las experiencias desde la subjetividad de los sujetos, de su mundo y desde nosotros como investigadores en formación. El enfoque nos permite recuperar las voces de aquellas niñas pertenecientes a la Casa Hogar "El Refugio de María" que poco a poco han sido ocultadas, dejando que se expresen y con ello, poder aproximarnos a su propia realidad. Con ello, presentamos un avance del marco teórico y metodológico de las experiencias de lectura, rescatando su voz y su representación del mundo, desde la perspectiva de Bolívar, Domingo y Fernández (2001), porque implicó rememorar aquellas huellas dejadas por los acontecimientos vividos y darles un sentido (Ricoeur, 2006).

Palabras clave: Experiencia de lectura, vulnerabilidad y lectura. 


\title{
Reconstruction of the reading experiences of vulnerable girls
}

\begin{abstract}
This article we present the reconstruction of the reading experiences of vulnerable girls who live in children's home in the city of Toluca considered as a "Refuge", given its social inequalities, this type of context in which girls live it affects the construction and reconstruction of themselves. And speaking of "himself", of his life, requires "establishing an order in the set of past events, between what was and is today, between past experiences and the appreciation they have acquired" (Bolívar, Domingo and Fernandez, 2001, p. 43). It is about weaving a written sequence of events that integrate their experiences with reading, in that sense; they will be able to tell stories from their own lives. For the research, we consider using the interpretive narrative approach that allows us, through the reconstruction of stories, to give an account of the research object. Bearing in mind that, through the narrative, we will give meaning to the experiences from the subjectivity of the subjects, their world and from us as researchers in training. The approach allows us to recover the voices of those girls belonging to the children's home "El Refugio de Maria" that little by little have been hidden, allowing them to express themselves and with it, to be able to approach their own reality. With this, we present an advance of the theoretical and methodological framework of reading experiences, rescuing their voice and their representation of the world, from the perspective of Bolívar, Domingo and Fernandez (2001), because it implied recalling those traces left by the events experienced and give them meaning (Ricoeur, 2006).
\end{abstract}

Keywords: Reading experience, vulnerability and reading.

\section{Introducción.}

El artículo es producto de las reflexiones hechas en el trabajo de investigación titulada Las marcas al leer. La lectura desde la experiencia de las niñas vulnerables de "El Refugio de María" como parte de mí formación del Programa de Doctorado en Ciencias de la Educación en el Instituto Superior de Ciencias de la Educación del Estado de México (ISCEEM).

El objeto de investigación es la producción de la experiencia como marca de las lecturas hechas por las niñas que tienen una condición de vulnerabilidad y que habitan en un Refugio 
considerado como hogar. El análisis se centra en conocer su vida lectora, sus encuentros y desencuentros con la lectura, los momentos en relación con su aprendizaje, las maneras de leer, además de escuchar sus sueños, deseos y su comprensión del mundo.

Resulta oportuno decir que empleamos el enfoque narrativo interpretativo, que es antes que todo, una perspectiva de investigación que y permite construir la realidad de forma narrativa. Así el proceso de construcción permitirá describir y contar los distintas experiencias, sucesos y acontecimientos. Hemos considerado una metodología biográfico narrativa que nos facilitará, comprender y " $<$ leer $>$ (en el sentido de interpretar) dichos hechos/acciones, a la luz de las historias que los agentes narran, se convierte en una perspectiva peculiar de investigación" (Bolívar, Domingo y Fernández, 2001, p. 10). Estableciendo como técnicas para recopilar la información: la observación, los diálogos informales, algunas notas de campo y la producción de las niñas.

En ese sentido, diremos que poner en evidencia las voces de las experiencias de lectura que fueron contadas en la investigación, tiene como propósito rescatar los relatos de las niñas como sujetos que viven una situación de vulnerabilidad y crisis dadas sus desigualdades sociales; y dar a conocer que tienen deseos, angustias e historias que las constituyen, las hacen ser lo que son y no solo verlas como sujetos investigados, sino desde su historicidad propia dadas sus condiciones.

El objetivo principal del trabajo es mostrar nuestro análisis e interpretación de las experiencias de lectura construidas por las niñas en una condición de vulnerabilidad que habitan en una Casa Hogar de la ciudad de Toluca. Con ello, pretendemos exteriorizar el proceso de producción de las experiencias lectoras de niñas que tienen una condición de vulnerabilidad.

En este tenor, la pregunta de investigación que guía el escrito es ¿Cómo producen sus experiencias de lectura las niñas que tienen una condición de vulnerabilidad y que habitan en una Casa Hogar considerada como "Refugio"? Ubicamos como categorías conceptuales a la experiencia de lectura, la vulnerabilidad y la lectura; desde referentes teóricos que nos parecen pertinentes como son Petit (2001, 2002, 2015), Larrosa (2003, 2006), Butler (2009, 2016), entre otros. 
En nuestra indagación del tema para la elaboración del estado de la cuestión nos percatamos que existen pocos trabajos respecto a las experiencias de lectura producidas por personas en condición de vulnerabilidad, específicamente con niñas pertenecientes a Casas Hogar. Eso nos llevó a interrogarnos acerca de lo que pasaba con la lectura de textos y sobre todo de su mundo, aquella que les permite constituirse. Por lo que podemos decir que nuestro objeto de investigación resulta importante, para mostrar aquel hecho a todos aquellos que nos encontramos en el campo educativo, retomando las palabras de Ricardo Sánchez (1993) es siempre complejo y existe la necesidad de indagar el estado de la cuestión para tomar decisiones en la investigación.

Podemos decir que al dar a conocer el análisis e interpretación de las experiencias relatadas por aquellas niñas pertenecientes a una Casa Hogar será una aportación diferente al campo de la cultura escrita, pues corresponde a espacios no convencionales que trabajan con la lectura. Así como de prácticas que se viven a diario en nuestro entorno más cercano; es decir, el mundo en el que nacemos es un "mundo intersubjetivo", donde definimos nuestra propia realidad al estar en constante interacción con los otros, por lo cual podemos decir que somos sujetos sociales por compartir un espacio y una lectura del mundo.

El artículo se encuentra estructurado en cinco apartados: El primero de ellos se refiere a las niñas como sujetos de experiencia que dada su condición de niñez se encuentran en vulnerabilidad, el segundo, nos permite adentrarnos el marco categorial que sustenta la investigación, en el tercero se alude a la perspectiva metodológica, en el cuarto compartimos algunas de las creaciones que se realizaron como parte del taller de lectura; en el quinto se proporciona una primera descripción de las experiencias de lectura de las niñas como parte de los hallazgos de la investigación, así como su análisis e interpretación; finalmente cerramos el artículo de manera temporal con algunas consideraciones.

\section{Las niñas de la Casa Hogar}

Reflexionar acerca de quiénes son las niñas de la Casa Hogar nos permite mirarlas como sujetos reales, de carne y hueso, con una historicidad porque han vivido situaciones de crisis y exclusión ante los demás; es decir, reconocerlas como sujetos vulnerables nos permite entender que lo son por su condición de infancia. La palabra vulnerabilidad adquiere un sentido distinto, se 
encuentra vinculada con la búsqueda por el reconocimiento, por ello al ser sentida, vivida y experimentada por los sujetos resulta ser subjetiva. La subjetividad infantil de las niñas se encuentra configurada en gran parte por su interacción con el mundo, por eso nos atrevemos a pensar que aquellas niñas heridas, dañadas o ignoradas socialmente, son vidas excluidas que les ha sido negada su existencia como sujetos constituidos y se les ha enmarcado sociablemente con estereotipos.

Desde este punto nos proponemos pensar a las niñas de la Casa Hogar como sujetos de experiencia que son aquellos que permiten que les pasen cosas, vulnerables y abiertos, como dice Jorge Larrosa (2003), que metafóricamente hablando son "un territorio de paso, de pasaje, algo así como una superficie de sensibilidad en la que lo que pasa afecta de algún modo, produce algunos afectos, inscribe algunas marcas, deja algunas huellas, algunos efectos” (p. 94).

Lejos de eludir la vinculación con la vulnerabilidad, trataremos de mostrar que son sujetos de experiencia porque se encuentran expuestas ante el mundo, vulnerables ante los sucesos viven. Así, podemos entender que su subjetividad es cambiante, está en un proceso de constitución, se va transformando dependiendo de las experiencias que las niñas mantienen con su propio contexto, es decir en El Refugio, en la escuela, con sus pares; se configuran en el mundo con sus contradicciones, incertidumbres, angustias, pasiones, con sus formas de ser y de comportarse.

Es por eso que vemos en ellas marcas distintas a las físicas (el maltrato y abuso sexual por el que han atravesado) porque les afectan el alma, trastocan su presencia frente a los demás. Visto de ese modo, las niñas son sujetos que actúan y construyen su entorno, producen experiencias y subjetividades, pero su acción tiene un sentido distinto a los adultos y es por eso que resultó necesario abrirnos a investigar esas nuevas vulnerabilidades que no han sido estudiadas señalar que deben ser tratadas desde nuevas perspectivas, pues nuestra realidad nos demuestra que la vulnerabilidad es una constante universal (Tello, 2016).

\section{Marco categoríal}

Contreras y Pérez (2010), consideran que cuando se investiga, lo importante radica en “encontrar los hilos del sentido", aquellos que nos guían a partir de nuestras vivencias y nos 
permiten ir encontrando un hilo del pensamiento. Así, la vulnerabilidad, la lectura y las experiencias de lectura serán los hilos que irán tejiendo nuestra investigación junto con la realidad.

Si bien, vulnerabilidad y lectura son dos palabras que parecen muy distantes, para Michéle Petit (2001) se encuentran relacionadas, al decirnos que la lectura es una buena aliada para reconstruir la subjetividad en situaciones de vulnerabilidad y el mundo interior se va modificando gracias al encuentro que se tiene con lo que leemos. Podemos pensar que la lectura posibilita esa reconstrucción después de atravesar alguna ruptura, un duelo, una pérdida. La lectura se puede considerar como un vehículo para que las niñas como sujetos vulnerables logren descubrirse, constituirse y leer su realidad que ya no está solo en las letras de un libro o un cuento; aquella que tiene ahora un matiz diferente.

La lectura como primer hilo conceptual requiere entenderla como parte de todos los contextos de nuestra vida, superando la idea de que el espacio escolar es el único desde donde se puede interactuar con ella. Resaltando que leemos el mundo antes que los textos, al respecto Freire (2008) nos dice:

La lectura del mundo precede a la lectura de la palabra, de ahí que la posterior lectura de ésta no pueda prescindir de la continuidad de la lectura de aquel. Lenguaje y realidad se vinculan dinámicamente. La comprensión del texto a ser alcanzada por su lectura crítica implica la percepción de relaciones entre el texto y el contexto (p. 94).

Así, "la lectura del mundo, del pequeño mundo", se da desde nuestra experiencia con lo cotidiano, desde nuestro contexto inmediato, que es parte fundamental para llegar a la "lectura de la palabra", porque el mundo necesita ser leído e interpretado; y la lectura exige entablar ese diálogo con el mundo, con el otro, para situarnos en dónde nos encontramos, implica recuerdos, canciones, poesías, películas, textos y contextos. O como dice Jorge Larrosa (2003)

(...) todo lo que nos pasa puede ser considerado un texto, algo que compromete nuestra capacidad de escucha, algo a lo que tenemos que prestar atención. Es como si los 
libros pero también las personas, los objetos, las obras de arte, la naturaleza, o los acontecimientos que suceden a nuestro alrededor quisieran decirnos algo (p. 29).

Entonces ¿qué implica leer? Implica no solo una apropiación de la significación, permite tomar una postura y una actitud; es mirar la lectura como un arte, crea experiencias no desde la mirada mecanicista. Su valor recae en la posibilidad para transformar nuestro pequeño mundo, dialogar y reflexionar a partir de situaciones vividas y provocando una experiencia en común con la lectura.

El segundo hilo conceptual, es la vulnerabilidad, entendida como aquella cualidad que tiene alguien para poder ser herido (Acosta, 2015), nos permite comprender que las niñas como sujetos vulnerables son aquel grupo que encuentra en una situación de riesgo, de ser herido por cualquier persona, dada su situación de infancia. La pena que sufren al vivir apartadas de sus familias, así como los cambios que ha conllevado todo eso, son acontecimientos que inquietan su mundo interior y las lleva a reconstruirse. Y considerarlas como sujetos vulnerables que requieren de asistencia, las coloca en ese proceso en el que se encuentran actualmente: de tutelaje e internamiento, separadas de su mundo.

Butler (2009) refiere que la vulnerabilidad se encuentra en estrecha relación con la pérdida y su relación "parece ser la consecuencia de nuestros cuerpos socialmente constituidos, sujetos a otros, amenazados por la pérdida, expuestos a otros y susceptibles de violencia a causa de esta exposición" (p. 46). Entendiendo que dependemos de otros y que cada uno de nosotros se constituye a partir de esa exposición.

El tercer hilo conceptual, las experiencias de lectura, aquellos acontecimientos que han marcado una manera de ver el mundo, aquellas que pueden darse de diversas maneras, citando las Larrosa (2003) quien considera que las experiencias se dan en forma de "shock, del choque, del estímulo, de la sensación pura, en la forma de la vivencia instantánea, puntual, desconectada” (p. 91) podemos ampliar la idea de la lectura, sobre todo la academicista, porque no solo es comprender, es vernos afectados con las palabras en un padecer. 
Para desarrollar el concepto de experiencia de lectura seguiremos a Larrosa (2003) quien nos dice

(...) la experiencia de la lectura no es desciframiento de un código sino construcción de sentido (...) el sentido de un texto estaría en las cosas que representa, en las ideas que transmite, en la voluntad del sujeto personal que la construye, en el contexto históricocultural en el que aparece o en los valores éticos o estéticos que encarna. En todos esos casos, el sentido estaría fuera del texto (aunque sería capturado a partir del texto). (p. 43).

Entonces es un fenómeno que no se puede controlar, por ello, depende totalmente de las oportunidades de acceso y los diversos espacios donde se desarrolla, así como de los lectores al dejar que algo les pase. En el caso de la lectura, podríamos pensar que es solo el sentido que le damos al texto, en relación con ello "la lectura no sería hacer que el texto asegurase su sentido en el mundo, sino hacer que el mundo suspenda por un instante su sentido y se abra a una posibilidad de resignificación" (Larrosa, 2006, p. 28). La experiencia de lectura no es el hecho de repetir los mismas ideas o conocimientos, implica pensar distinto de cómo se piensa y percibir distinto de cómo se ve.

\section{El entramado metodológico}

El presente apartado, nos permite mostrar el desarrollo de las estrategias metodológicas necesarias para recuperar los diferentes diálogos, la escritura y los diversos momentos que se vivieron en el trabajo de campo que fueron entramando la reflexión en el camino de la investigación. Por ello, para conocer las experiencias que han ido dejando los libros en la ruta de vida de las niñas consideramos el método biográfico narrativo que otorga significado a lo vivido mediante un proceso de reflexión, más que un método de investigación es "un proceso dialógico" (Bolívar, Domingo y Fernández, 2001), dejando que expresen su experiencia y con ello, poder aproximarnos a su realidad. Por ello, el diálogo como medio posibilitó la reflexión del relato, es

decir, nuestras conversaciones se fueron poco a poco transformando en una historia, como dice Arfuch (1995) en "una puesta de sentido. La narración no es una mera <representación> de lo ocurrido, sino una forma que lo hace inteligible, una construcción que postula relaciones que quizá no existen en otro lugar, casualidades, causalidades, interpretaciones" (p. 100). 
Y la narrativa como aquel tejido de historias en donde se mezclan acontecimientos vivencias y experiencias necesita de apoyarse de diversas técnicas para obtener datos sobre la experiencia de ciertas personas para describirlas y analizarlas, así, los relatos, las notas de campo, los diálogos informales, los escritos propios permitieron relatar y contar sus historias para extraer lo valioso que se desarrolla más adelante en los hallazgos. Lo cual nos llevó a explorar e indagar una realidad y situarnos en la escuela primaria "Justo Sierra Méndez" ubicado en la zona centro de la ciudad de Toluca. El escenario para construir y vivir esas historias fue un Taller de lectura, en él contamos historias, encaminadas a soñar, navegar y viajar en medio de palabras, creamos un espacio de cuentos leídos en voz alta que consideramos les pueden ayudar a formular su propia historia, su propio sentido de la vida, y de manera implícita "sugiriendo que la vida tiene una dirección, un sentido, aun cuando haya rupturas" (Petit, 2015, p. 91). Y entre cuentos, poemas e historias narradas nos encaminamos a conocer en lo cotidiano, aquello que justamente buscamos: "sus experiencias con la lectura".

El Taller de lectura estaba dirigido a aquellas niñas en condición de vulnerabilidad que pertenecen a la Casa Hogar denominada "El Refugio de María", el grupo se conformó por doce niñas de segundo a sexto grado con una edad entre 7 y 14 años. Partimos de distintos ejes de análisis como son: el aprendizaje de la lectura, elección de la lectura, tiempos y espacios para leer, entre otros. Se vivió en dos momentos: el primer momento que fue a manera de exploración en los meses de abril y mayo del ciclo escolar 2019-2020 y un segundo momento que comprendió de octubre a marzo del ciclo escolar 2020-2021.

\section{"Déjenos leer". EI taller de lectura}

El Taller de lectura lo denominamos "Déjenos leer" creado a partir de los objetivos de investigación que permitió viviéramos nuestra propia experiencia, al ser contadores de cuentos y construimos junto con las niñas una misma historia encaminada a soñar, navegar y viajar en medio de palabras, así en medio de cuentos que permitieron conocerlas en lo cotidiano creamos un espacio de sentidos. Por eso, nos parece importante compartir algunas de las creaciones que se realizaron en el taller, las agrupamos de acuerdo al tipo de expresión-creación que se manifestó después de que les leíamos historias, aquí hablaremos solo de tres. 
Una de los primeras expresiones que nombramos "Dibujando una vida" se refiere a la realización de dibujos aquellos en donde ellas eran las protagonistas de su relato. Su dibujo pegado en la pared se hacia visible para todas y con ello también su sentir, de acuerdo con María López (2016) aquello les permite "una afirmación de su lugar en el mundo, sentir una extensión de sí mismo, una confirmación de que es posible crear vida, y quien crea vida puede demostrar que está vivo" (p. 63). Aunque los trazos de un dibujo, no solo mostraron sus experiencias con la lectura, también su actitud, por ejemplo, Jocelyn en las actividades del taller tomaba las hojas con enojo, azotándolas sobre su pupitre, entendimos con el paso de las sesiones que no sabía leer y escribir de manera convencional y aprovechaba el espacio del taller para acercarse a nosotros, cuando leíamos buscaba la forma de tocar nuestra mano o el libro que leíamos. Nunca estaba sentada, siempre caminaba por todo el espacio, mirando a sus compañeras y esperando una reacción de ellas y de nosotros. Por eso, pensamos que todos los dibujos que realizó y que terminaron arrugados, sucios o hechos varias veces eran parte de una voz que quería que ser reconocida.

Otra forma de expresión fue "Modelando la subjetividad", fue un proceso de creación de figuras con arcilla, en ella pudimos mirar aquello que no tan fácilmente se dejó ver en los dibujos, eso que sin palabras conectó en una de ellas su necesidad de amor fraternal. María López (2016) dice que debemos ofrecer materiales, estímulos; pero que no habría una posibilidad de construcción imaginaria sin el otro ser, en este caso de las niñas y que por medio de esos materiales que ayudaron a organizar las vivencias, les permite reconocerse y les ofrecen una riqueza cultural. Por ejemplo Carolina que trabajo con delicadeza la arcilla, elaboró la figura de una madre abrazando a un bebé, envueltos ambos con arcilla, formando un solo cuerpo, así, sin pies, ni manos, formó esa figura, pero no solo era una escultura de una madre, era la de su propia mamá, la cual había representado con ayuda de formas y diseños.

Otra creación fue la de “Mi máscara” que nos ayudó a descifrar esas palabras no dichas, a no obviar sus manifestaciones y actitudes que narraban también su historia expresada como dice Anzaldua (2004) de forma metafórica, con el fin de que el deseo y las significaciones imaginarias despierten la imagen que guardan de su propia infancia y sobre todo "constituyen una técnica proyectiva muy poderosa, de tal manera que estas están conformadas de significaciones 
imaginarias y elementos subjetivos vinculados a efectos de deseo, fantasía y elementos de identificación" (p. 158).

Para iniciar la actividad se les pidió que elaboraran una máscara de león, no les dimos más indicaciones y les entregamos una bolsa de papel, papeles de colores, hojas de color, tijeras, pegamento y plumones. Paola, una de las niñas más grandes, le dio mayor énfasis a la forma de los ojos, los hizo ovalados con cuadros de color y los ordeno de acuerdo a una secuencia (amarillo, rosa y naranja). La boca en su máscara pareció no importarle, no se la coloco, ni siquiera trazada con bolígrafo como algunas otras niñas lo hicieron. Quizá, aquella boca que no tenía la máscara y que pareciera irrelevante, nos hable de ese silencio, de aquel mundo que aparta de la vista de los demás, eso que ha marcado su infancia como un secreto, como dice Martín Garzo (2015) "permanece un reino de silencio donde se habla el lenguaje de las cosas mudas (...), ese reino mudo es el reino de la infancia que significa literalmente una incapacidad de hablar" (p. 49).

La expresión artística, estuvo presente en casi todos nuestros encuentros, porque lo que no se decía con palabras, se expresaba con sus creaciones, de modo que, crear, permitió ver en ellas lo no visible, lo que hablaba por ellas, la narración de su vida contada con otro tipo de lenguaje. Nos permitimos incluirnos en su mundo, nos conducimos a ese lugar donde habitan sus experiencias, alegrías, dolores y angustias y que a veces eran difíciles de contar con palabras. Era como dice Andruetto (2014) "ir en busca de una lengua única hecha con la lengua de todos [era] permanecer alertas, como los pescadores, pero con una red hecha de palabras [diremos nosotros: de creaciones]" (p. 51).

\section{Las experiencias de lectura}

Para dar paso a las construcciones narrativas diremos que, de las doce niñas, consideramos solo cinco casos particulares, de los cuales solo hablaremos aquí de dos. Para su selección nos basamos en varias situaciones: en primer lugar, por el hecho de que algunas no han consolidado la lectura y escritura convencional, en segundo, porque observamos un mayor apego hacia la lectura, escritura y el arte; y en tercero, porque consideramos que era necesario compartir esas historias, sin menospreciar la de sus compañeras. Los relatos fueron elaborados a partir de los registros, dibujos, creaciones, y observaciones que recuperamos durante el taller. 


\section{La experiencia de lectura de Yarely}

Yarely es una niña que no sabe leer, dicho desde las ideas convencionales de lectura que consideran que es el proceso de descifrar códigos escritos o como habilidad para realizar actividades escolares, sin embargo, la lectura, es más que eso, es la construcción de un sentido, no solo desde las letras también desde el lugar en que se vive; Graciela Montes (2017) define la lectura "como la conducta social por la cual las personas nos apropiamos de algunos discursos significantes (o sea, parte de nuestra cultura) de la sociedad en que vivimos". De esa manera Yarely, lee y se apropia de su cultura, aquella que va desde una imagen, un lugar, un espacio, una experiencia, desde su propia realidad.

Para ella, el no saber leer textos no ha sido un impedimento para que haga experiencias, En una de las sesiones le pidió ayuda a su hermana para que escribiera por ella, en el diálogo posterior que compartió con nosotros nos relató lo siguiente: "No me gusta leer, pero me encantaría leer, me gusta el libro de los puerquitos, y mi experiencia es buena, me encantaría leer... sí quiero, aunque no lo intento, porque tengo miedo a que se burlen de mí. Me gusta leer libros cortos" (Yarely, Trabajo de campo, 2019)

En su dibujo del lado inferior podemos encontrar parte de sus emociones para con la lectura, ya que dibuja un libro abierto y junto al mismo una cara triste, que nos deja ver que se siente así porque ella desea leer cuando reafirma en el escrito "me encantaría leer". Respecto a ello Bettelheim y Zelan (2001) nos dicen que para que el niño desee aprender a leer no es necesario que la lectura sea vista desde su utilidad, sino desde una firme creencia de que "saber leer abrirá ante él un mundo de experiencias maravillosas, le permitirá despojarse de su ignorancia, comprender el mundo y ser dueño de su destino" (p. 56).

Ante ello, la experiencia de la lectura es otra cosa que solo comprender; es cierto que cuando socializamos una narración hablamos de eso, pero siempre para cotejarlo con las experiencias propias. Leemos para conocer el mundo, también se lee como un mecanismo de evasión de nuestro mundo real, como dice Petit (2002) la lectura es una posibilidad para entrar a un mundo, aquel en el que se suspende por un instante el nuestro y adopta un espacio propio. 
Otra de las cosas que notamos en Yarely a diferencia de sus compañeras fue el querer vernos como una figura materna, en varias sesiones nos dijo mamá de manera natural, no como equivocación, sino como una manera de manifestar su pérdida maternal; si bien no le dimos importancia en su momento, porque parecía una equivocación, el sentimiento que tuvimos después se convirtió en tristeza, imaginamos de pronto las propias personas cercanas a nosotros y sentimos también su dolor, al entender que a esa corta edad ha experimentado situaciones de angustia fuera del alcance de sus manos. En uno de sus escritos a modo de carta que realizó con ayuda de una compañera dejó ver aquel cariño faltante en su vida. La carta dice así:

Mamá esta carta es para ti, espero que estés feliz con esta carta te extraño Estoy triste por no conocerte, quería conocerte y vivir momentos felices contigo.

\section{TE AMO mamá}

Yarely (Yarely, Trabajo de campo, 2019)

Reconocemos en la voz escrita, que se siente triste y añora ser feliz, imagina una familia, una mamá, lo hace posible en su imaginación, como lo dicen Córdoba y Machado (2016) "Todo huérfano, en la realidad y en la literatura, ya lo vimos, tiene padres en su imaginación" (p. 34). Con el paso de las sesiones, nos dimos cuenta que ella comenzó a escribir palabras, un poco a leer, y a mostrar mucho interés por adentrarse a las historias, dicho por su maestra ha ido cambiando poco a poco. Entendemos que fuera del lenguaje escrito esta su mundo, aquel que comparte mediante un vínculo afectivo.

\section{La experiencia de lectura de Carolina}

Cuando se leyó en voz alta el poema de Mario Benedetti "No te rindas" y se les propuso que hicieran un dibujo de lo que les hizo sentir el poema y escribir un poema de forma posterior. Carolina, una niña de 14 años, que llegó al Refugio desde los cuatro siempre mostró un interés distinto al de sus compañeras por leer libros. Uno de los versos que escribió dice así:

\section{Duele perder un amigo}




\section{Dejándome un regalito}

\section{Que se va de rápido}

Sin decirme adiosito (Carolina, Trabajo de campo, 2019)

En el poema, tal vez nos parezca muy simples sus palabras, sin embargo, está dirigido a una de sus amigas con las que comparten su vida en el "Refugio", no es actual, lo escribió un año antes cuando aquella persona partió de ese lugar, pero lo recordó en esos momentos y lo escribió. Hay una experiencia de ruptura, de desfamiliarización con alguien que afectó su sensibilidad, sus emociones, su deseo, para Butler (2016), esas rupturas se instalan en el presente como una incapacidad de dar cuenta de nosotros mismos por estar sometida por otros, por otras, por el "Refugio" y por el vínculo que tiene con todo aquello que le ha rodeado desde su más tierna infancia.

Podemos pensar, que aquella compañera que se ha ido y que formó parte de su grupo de amigas era la misma con la que compartió historias antes de dormir. En uno de sus relatos nos dijo que había una compañera que les contaba cuentos por las noches, los inventaba y se los compartía, aunque ya no vive en El Refugio, ella, aún la recuerda. Probablemente esas lecturas contadas antes de dormir le habían ayudado a superar sus miedos, a trazar un camino más allá de su propio mundo, como refiere Petit (2015) los libros leídos antes de dormir en voz alta presentan el mundo de otro y permiten atravesar la noche para no tener miedo al abandono, a lo desconocido y con ellos escribimos una historia propia entre las líneas leídas.

Y es que la sensibilidad que muestra Carolina en sus poemas, se puede ver también en los trazos de sus dibujos, aquellos destacaron de los del resto de sus compañeras, la mayoría eran hechos a lápiz y trazados delicadamente, los rasgos faciales eran muy finos, se tomaba su tiempo para delinearlos, una característica de estos siempre fueron los ojos, dejaban ver aquellas emociones que sentía en ese día, algunas veces los hizo cerrados, otros abiertos, pero siempre muy expresivos. Uno de ellos, representó su relación con la lectura, y que frente al texto y el acompañamiento de la religiosa al tocar su hombro mientras el libro abierto le incitaba a leer, dejando ver que existe un acercamiento a través de la lectura sin obligación. 
Bettelheim y Zelan (2001) nos dice que "la mayoría de la gente solo lee cuando realmente le interesa lo que está leyendo, todos los esfuerzos, desde el mismo principio de la enseñanza de la lectura, deberían ir dirigido a ese objetivo" (p. 44), y eso cobra sentido, cuando vemos el gusto por Carolina llevarse el libro de "Leyendas japonesas", y mostrándose continuamente interesada por cada uno de sus cuentos:

Con el de leyendas japonesas, me daban mucha tensión sobre saber más de sus historias, sus leyendas, me gustó la leyenda La mujer de la neblina, en el que la chica era muy hermosa y siempre estaba orgullosa (Carolina, Trabajo de campo, 2019).

Dicho por sus compañeras, le gusta leer en "El Refugio", pero ha ido perdiendo interés en la escuela. Algo que también su maestra señala, se ha vuelto rebelde y contesta mal, desde hace mucho ya no platica con ninguna de sus compañeras, se aísla y se molesta con todos y todas. El arte la hace feliz, se refugia en sus dibujos, en sus escritos, en su libreta que es como una compañera para ella, no sabemos si la partida de esa persona a la que le dedica el poema sea la causa de su comportamiento, o si sus dibujos nos cuenten otra historia que la ha hecho distante, diferente ante los ojos de los demás.

\section{Consideraciones finales}

Podemos señalar que el avance presentado hasta el momento en la investigación, nos permite hacer visible las experiencias de lectura que tienen las niñas en condición de vulnerabilidad; así mismo, las categorías teóricas nos han permitido analizar el fenómeno investigado. Sin embargo, la investigación también nos permitió tener una "experiencia singular" con las niñas por tener la oportunidad de conocer su historia, entender su desigualdad, comprender su agresividad y sentir su soledad; porque su vida y la nuestra están entretejidas con un sinnúmero de acontecimientos, vivencias, interacciones con el otro que se entrelazan con nuestras maneras de sentir, pensar y hacer. En esos espacios que la investigación posibilita nos permitimos ir tejiendo la escritura de sus narrativas y a través de ellas darle sentido a nuestra vida, porque los distintos encuentros que vivimos en la investigación nos invitaron a reflexionar acerca de nuestras propias pérdidas, lecturas y experiencias que han acontecido. 
Así, la narrativa de las niñas nos muestra que leer no es solo un asunto de textos, sino una manera de "leer el mundo", que implica un modo de estar en él, sus maneras de hacer y de hacerse dentro de él (Espinosa, 2013, p. 19); pues ellas muestran narran esas maneras de comprender y habitar en el mundo de distintas formas. Esa reflexión nos invita a pensar que las experiencias de lectura trascienden de la lectura de textos, se viven en y con el contexto en el que se vive e interactúa.

Autor: Salgado, Adriana ${ }^{1}$.

\section{Referencias bibliográficas}

Acosta, F. (2015). Red de contención infantil como modelo de intervención en situaciones de vulnerabilidad infantil. Buenos Aires: CLACSO. Recuperado de http://biblioteca.clacso.edu.ar/clacso/posgrados/20151221013950/ACOSTA67.pdf

Andruetto, M. (2014). La lectura, otra revolución. México: Fondo de Cultura Económica.

Anzaldúa, R. (2004). La docencia frente al espejo: imaginario, transferencia y poder. México: Universidad Autónoma Metropolitana

Arfuch, L. (1995). La entrevista, una invención dialógica. España: Paidós.

Bettelheim, B. y Zelan, K. (2001). Aprender a leer. Barcelona: Crítica.

Bolívar, A., Domingo, J. y Fernández, M. (2001). La investigación biográfico-narrativa en educación. Enfoque y metodología. Madrid: Murallas S. A.

Butler, J. (2009). Vida precaria. El poder del duelo y la violencia. Buenos Aires: Paidós.

Butler, J. (2016). Los sentidos del sujeto. Barcelona: Herder

Contreras, J., y Pérez de Lara, N. (2010). Investigar la experiencia educativa. Madrid: Morata.

\footnotetext{
${ }^{1}$ Egresada del programa de Doctorado en Ciencias de la Educación del Instituto Superior de Ciencias de la Educación del Estado de México. Promoción 2018-2020.
} 
Córdoba, A., y Machado, A. (2016). La familia en la literatura infantil. Imaginación y realidad. México: Secretaria de Cultura.

Espinosa, A. (2013). Configuración de la subjetividad en la primera infancia en un mundo posmoderno. Revista Infancia imágenes, 12(2), 18-28.

Freire, P. (2008). La importancia de leer y el proceso de liberación. México: Siglo Veintiuno Editores.

Garzo, M. (2015). Lo real ¿dónde está? La literatura como fascinación. México: Secretaria de Cultura.

Larrosa, J. (2003). La experiencia de la lectura Estudios sobre literatura y formación. México: Fondo de Cultura Económica.

Larrosa, J. (2006). Sobre la experiencia. Aloma. Revista de Psicología i Ciències de l'Educaciò (19) 87-112. Recuperado de https://hdl.handle.net/2445/96984.

López, M. (2016). Un mundo abierto. Cultura y primera infancia. México: Secretaria de Cultura.

Montes, G. (2017). La frontera indómita. En torno a la construcción y defensa del espacio poético. México: Fondo de Cultura Económica.

Petit, M. (2001). Lecturas: del espacio íntimo al espacio público. México: Fondo de Cultura Económica.

Petit, M. (2002). Pero ¿y qué buscan nuestros niños en sus libros? México: Secretaria de Cultura.

Petit, M. (2015). Leer el mundo Experiencias actuales de transmisión cultural. Argentina: Fondo de Cultura Económica.

Ricoeur, P. (2006). La vida: en busca de un narrador. Revista Ágora, 25(2), 9-22.

Sánchez, R. (1993). Didáctica de la problematización en el campo científico de la educación. Perfiles Educativos (61), 64-78. 
Tello, L. (2016). Derechos Humanos y vulnerabilidad. En Pérez, M., Macías, Ma. Carmen., González, N., y Rodríguez S. Temas selectos de Vulnerabilidad y violencia contra niños, niñas y adolescentes. México: Universidad Nacional Autónoma de México. Instituto de investigaciones jurídicas. 